J Medicine 2018; 19: 18-21

\title{
Requirement of Intravenous Fluid and Insulin in the Management of Diabetic Ketoacidosis to Overcome the Crisis: Experience in a Specialized Hospital
}

\author{
ROZANA ROUF, ${ }^{1}$ MURAD RAHIM, ${ }^{2}$ ZAFAR AHMED LATIF ${ }^{3}$
}

\begin{abstract}
Background: Among the acute metabolic decompensation states of diabetes mellitus, diabetic ketoacidosis (DKA) is relatively common. It commonly occurs in diabetic patients who have given up taking insulin or who have got some sort of infection. Treatment consisted of correction of dehydration, hyperglycaemia, electrolyte imbalance, acidosis and antibiotics for the patients who have some sort of infection or treatment of other precipitating cause, if present.

Methods: This cross sectional study was performed in BIRDEM Hospital in 2012 on fifty adult diabetic ketoacidosis patients who fulfilled inclusion and exclusion criteria to find out the total amount of intravenous fluid (normal saline) needed to treat diabetic ketoacidosis.

Results: DKA occurred more in previously diagnosed (60\%) diabetic patients and frequency was more in poor, village people and there was no significant sex differences. Along with polyuria (24\%) and polydipsia (16\%), nausea (32\%), vomiting (48\%), abdominal pain (28\%) and feature of infection were common. Infection (38\%) and omission of insulin doses (34\%) were the main cause. The glycaemic status was poor in most cases. Most patients had mild (32\%) to moderate (60\%) acidosis and gross electrolyte disturbance was not that much common. Leukocytosis (88\%) was invariably present, even without any infection. Outcome ( $90 \%$ survival) was comparable with developing world. In management of DKA 40\% patients required 11 liters of fluid, who had increased diuresis. 24\% patients required 91-100 units of insulin, and most (32\%) patients took 48 hours for correction of acidosis.
\end{abstract}

Conclusions: Diagnosis and treatment of diabetic ketoacidosis is not difficult if recognized early. Adequate fluid replacement is important to prevent the mortality.

Keywords: Diabetes Mellitus, Diabetic Ketoacidosis, Insulin, Intravenous Fluid.

\section{Introduction:}

Among the acute metabolic decompensated states of diabetes mellitus, diabetic ketoacidosis is relatively common. It commonly occurs in diabetic patients who have given up taking insulin or who have got some sort of infection. Previously undiagnosed patients also presented with DKA. For this reason, we have evaluated fifty DKA patients to see the requirement of I/V fluid, and insulin in the management and the outcome. Treatment consisted of correction of dehydration, acidosis and electrolyte imbalance and also treatment of precipitating factor eg. antibiotic for infection. ${ }^{1,2}$

1. Dept. of Endocrinology, BIRDEM, Dhaka.

2. Asst. Prof., Dept. of Nephrology, BIRDEM, Dhaka.

3. Prof. of Endocrinology and Academic Director, BIRDEM, Dhaka.

Corresponding author: Dr. Rozana Rouf, Dept. of Endocrinology, BIRDEM, Dhaka. E-mail: rozanarouf@gmail.com.

\section{Methods:}

This was a cross sectional study in Department of Endocrinology, BIRDEM, Dhaka from March, 2012 to March, 2012. Portland protocol was followed for the management of DKA. Blood sugar was measured hourly and rate of insulin was adjusted in syringe pump according to the Portland protocol. Intake-output chart was maintained to see whether $50 \%$ of infused fluid is retained and also to prevent volume overload. Bicarbonate was not given. DKA was considered resolved when bicarbonate was above $18 \mathrm{meq} / \mathrm{l}$ and $\mathrm{pH}>7.3$. Then split mix regimen of subcutaneous insulin was started when patients could take food orally (2/3 intermediate acting NPH insulin and $1 / 3$ as regular insulin). $2 / 3$ of the total daily dose was given in the morning and $1 / 3$ in the evening.

\section{Results:}

Total no of patients were 50 . Newly diagnosed diabetic case was 20 and 30 known diabetic. Among the known cases 50\% patients were on insulin, 25\% were on OHA followed by insulin and 22\% were on OHA. 20 cases were male and 30 
were female. (M:F=2:3). Infection and omission of insulin doses were the main causes of developing DKA. The glycaemic status was poor in most cases. Most patients had mild to moderate acidosis. In management of DKA $40 \%$ patients required 11 liters of fluid, who had increased diuresis. Twenty four present patients required 91-100 units of insulin, and most (32\%) patients took 48 hours for correction of acidosis. Table 1 describes the distribution of patients of different age groups. $72 \%$ patients were of below 40 years, while only 1 patient $(2 \%)$ was above 50 years. More precisely, maximum $24 \%$ patients were in between 21 and 25 years of age.

\section{Table-I}

Age distribution of patients (total patients 50)

\begin{tabular}{cc}
\hline Age (years) & Number of patients (\%) \\
\hline$\leq 20$ & $8(16)$ \\
$21-25$ & $12(24)$ \\
$26-30$ & $7(14)$ \\
$31-35$ & $4(8)$ \\
$36-40$ & $5(10)$ \\
$41-45$ & $9(18)$ \\
$46-50$ & $4(8)$ \\
$51-55$ & $1(2)$ \\
$56-60$ & $0(0)$ \\
$\geq 61$ & $0(0)$ \\
\hline
\end{tabular}

Table-II

Distribution of cases according to precipitating causes

\begin{tabular}{lcc}
\hline Cause & Number of patients (old) & Number of cases (new) \\
\hline $\begin{array}{l}\text { Infection (38\%) } \\
\text { Omission of }\end{array}$ & 10 & 9 \\
insulin/ drug (34\%) & 17 & 10 \\
New case (20\%) & & \\
Surgery (2\%) & 1 & 1 \\
Pancreatitis (6\%) & 2 & \\
\hline
\end{tabular}

Table-III

RBS levels at presentation

\begin{tabular}{ccc}
\hline RBS mmol/1 & Number of patients & Percentage \\
\hline$\leq 10$ & 0 & 0 \\
$10-15$ & 1 & 2 \\
$16-20$ & 7 & 14 \\
$21-25$ & 19 & 19 \\
$26-30$ & 9 & 18 \\
$31-35$ & 6 & 12 \\
$>35$ & 8 & 16 \\
\hline
\end{tabular}

Table-IV

Degree of acidosis of patients

\begin{tabular}{lcc}
\hline $\mathrm{pH}$ & Number of patients & Percentage \\
\hline$<7$ & 4 & 8 \\
$7-7.24$ & 27 & 54 \\
$7.25-7.30$ & 19 & 38 \\
\hline
\end{tabular}

Table-V

Requirement of fluid for correction of acidosis

\begin{tabular}{cc}
\hline Amount of (NS) fluid (L) & Number of patient \\
\hline 5 & 9 \\
6 & 4 \\
7 & 1 \\
8 & 4 \\
9 & 2 \\
10 & 5 \\
11 & 20 \\
12 & 2 \\
13 & 2 \\
14 & 2 \\
16 & 2
\end{tabular}


Table-VI

Requirement of insulin for correction of acidosis

\begin{tabular}{cc}
\hline Amount (unit) & Number of patient \\
\hline$<80$ & 10 \\
$81-90$ & 8 \\
$91-100$ & $12(24 \%)$ \\
$101-110$ & 3 \\
$111-120$ & 4 \\
$121-130$ & 4 \\
$131-140$ & 6 \\
$141-150$ & 1 \\
$151-160$ & 2 \\
\hline
\end{tabular}

Table-VII

Time taken for correction of acidosis

\begin{tabular}{cc}
\hline Time & Number of patients \\
\hline $24 \mathrm{hrs}$ & 10 \\
$48 \mathrm{hrs}$ & $16(32 \%)$ \\
$72 \mathrm{hrs}$ & 8 \\
4 days & 2 \\
5 days & 2 \\
6 days & 1 \\
7 days & 1 \\
\hline
\end{tabular}

\section{Discussion:}

This study was done to see the requirement of intravenous fluid and insulin in the management of DKA to overcome the crisis, which was experienced in a specialized hospital, BIRDEM. Female: male ratio in Denmark ${ }^{1}$ was $7.2: 5.7$. In a recent study in Taipei, ${ }^{2}$ it was found that $67 \%$ DKA patients were female. In a small series in India ${ }^{3}$ male: female ratio was 2:1. The patients of mild to moderate acidosis was treated in general ward as it was recommended in a study at Emory University School of Medicine, Atlanta. ${ }^{4}$

Infection was the commonest (36\%) precipitating cause in this study. Among the new 20 cases, 9 had documented infection. Possibly they had previously undiagnosed hyperglycaemia and infection had precipitated DKA. The remaining 10 patients had a relatively short history of polyuria, polydypsia, weight loss and rapid breathing, 1 patient had acute pancreatitis. Of the 30 diagnosed cases, 17 (34\%) patients had omitted insulin and they developed DKA in 1-18 days. 10 cases had infection, 2 cases had acute pancreatitis. In one case DKA was precipitated by omitting insulin after gynaecological operation.

Most patients had normal or slightly low $\mathrm{Na}+$ level, high normal or slightly elevated $\mathrm{K}+$ levels. Similar results were found in a study in Pakistan ${ }^{5}$ and at Atlanta. ${ }^{4}$ Four patients had severe hyponatraemia, hypokalaemia and severe acidosis. After initiation of treatment with fluid and insulin, almost all patients developed hypokalaemia requiring intravenous correction. In a national survey of Denmark ${ }^{6}$ similar observation appeared.

Most patients had grossly elevated blood sugar levels and ++ or more ketonuria on urine ketostix test. Patients were found to have ketonuria for longer period in comparison with their clinical and biochemical improvement evidenced by $\mathrm{pH}$ and HCO3- levels. Similar observation is reported in a small study in India ${ }^{2}$ very recently. Most of the patients (24\%) took $48 \mathrm{hrs}$ for correction of acidosis and maximum time taken was 7 days. In the study at Emroy University School of Medicine time taken was 11 to 14 hours. ${ }^{6}$

The outcome was quiet satisfactory and comparable with developed countries. $5(10 \%)$ patients expired and these patients had severe acidosis. Mortality was $6-24 \%$ in developing country. ${ }^{7}$ In our study most of the patients needed 91-100 unit of insulin which was similar with a study done at Emroy University School of Medicine ${ }^{4}$ and it was 82-110 unit of insulin. During the first 24 hours of in-hospital treatment, each patient required, on an average of 4.12 litres of intravenous fluid, $60 \mathrm{mmol}$ of potassium and 72 units of insulin. ${ }^{8}$

\section{Conclusions:}

DKA occurred more commonly in known diabetic patients who have omitted insulin doses or have had infection. New cases are not uncommon. Polyuria, polydypsia, vomiting, abdominal pain and infection are common features. The glycaemic control in these patients is poor. Severe acidosis is less common. In mild to moderate acidosis, gross electrolyte disturbances are infrequent but hypokalaemia develops after initiation of treatment. Leukocytosis is common, even in absence of infection and ketonuria may persist or even increase with treatment in spite of clinical 
JM Vol. 19, No. 1 Requirement of Intravenous Fluid and Insulin in the Management of Diabetic Ketoacidosis to overcome the Crisis: Experience in a Specialized Hospital

and biochemical improvement. The overall outcome is good and mortality rate is $10 \%$.

\section{Conflict of interest: None.}

\section{References:}

1. Henriksen OM, Roder ME, Prahl JB, Svendsen OL. Diabetic ketoacidosis in Denmark: incidence and mortality from public health registries. Diabetes Res Clin Pract. 2007;76 (1):51-6.

2. Lin SF, Lin JD, Huang YY. Diabetic ketoacidosis: comparisons of patient characteristics, clinical presentations and outcomes today and 20 years ago. Chang Gunh Med J 2005;28(1):24-30.

3. Jahagirdar RR, Khadilkar VV, Khadilkar AV, Lalwani SK. Management of diabetic ketoacidosis in PICU. Indian J Pediatr 2007;74(6):551-4.

4. Umpierrez GE, Cuervo R, Karabell A, Latif K, Amado X Freire and Abbas E. Treatment of DKA with I/V insulin. Diabetes Care 2004(8):1873-1878.

5. Jabbar A, Farooqui K, Habib A, Islam N, Haque N, Akhter J. Clinical characteristics and outcomes of diabetic ketoacidosis in Pakistani adults with type 2 diabetes mellitus. Diabet Med. 2004;21(8):920-3.

6. Henriksen OM, Prahl JB, Roder ME, Svendsen OL. Treatment of diabetic ketoacidosis in adults in Denmark: a national survey. Diabetes Res Clin Pract. 2007;77(1):113-9.

7. H.E. Lebovitz. Diabetic Ketoacidosis. The Lancet, 1995;345(8952):762-772.

8. Rahim MA, Uddin KN, Zaman S, Musa AKM, Rahman MR, Hossain MD, Ahmed AKMS. Clinical Spectrum and managemet of DKA: Experience in a tertiary care hospital. Birdem Medical Journal. 2011;1(1). 